PROCEEDINGS OF THE

AMERICAN MATHEMATICAL SOCIETY

Volume 98, Number 2, October 1986

\title{
GENERALIZED REFINABLE MAPS
}

\author{
E. E. GRACE
}

ABSTRACT. Relationships among refinability, two successively greater generalizations, and a related property are studied, for maps between compacta.

\section{Introduction.}

Definition. A mapping $f$ from a compactum $X$ onto a compactum $Y$ is refinable if, for each positive number $\varepsilon$, there is an $\varepsilon$-mapping $g$, from $X$ onto $Y$, such that $d(f(x), g(x))<\varepsilon$, for each $x$ in $X$.

In considering what properties of refinable maps [1, p. 263] are essential to their preservation of the proximate fixed point property, two successively more general classes of maps, proximately refinable maps and weakly refinable maps, presented themselves. Maps of these types (and closely related proximate near homeomorphisms) preserve the proximate fixed point property [3]. There are interesting relationships among these maps and refinable maps, and additional interesting properties of these maps, which are presented here. In particular, it is shown that a proximately refinable map onto a continuous curve is monotone, and that is used to show that weakly refinable maps preserve the property of being a graph.

It is known [2, Corollary 1, p. 142], that a map defined on a graph is refinable if and only if it is a near homeomorphism. In [4] it is shown that the composite of proximately refinable maps is proximately refinable, and that is used to show that a map defined on a graph is proximately refinable if and only if it is monotone. Also in [4], proximately refinable maps are shown to have some related properties if the domains have certain kinds of decompositions into graphs.

\section{Definitions and notation.}

Notation. $X$ and $Y$ are compacta, i.e., compact metric spaces. $f: X \rightarrow Y$ means $f$ is a function from $X$ into $Y$, and $f: X \rightarrow Y$ means $f$ is a function from $X$ on to $Y$.

Definitions. A function $g$ into a metric space is $\varepsilon$-continuous if, for each $x$ in the domain of $g$, there is a neighborhood $D$ of $x$ such that $g[D] \subseteq N_{\varepsilon}(g(x))$, i.e., such that $d\left(g(x), g\left(x_{1}\right)\right)<\varepsilon$, for each $x_{1}$ in $D$. A map is a continuous function (but the verb does not connote continuity). $f: X \rightarrow Y$ is an $\varepsilon$-function if $\operatorname{diam}\left(f^{-1}(y)\right)<\varepsilon$,

Received by the editors June 28, 1985. Presented to the Society, January 10, 1986.

1980 Mathematics Subject Classification. Primary 54C10; Secondary 54E45, 54G20.

Key words and phrases. Refinable map, proximately refinable map, weakly refinable map, proximate near homeomorphism, compactum. 
for every $y$ in $Y$, 'and is a strong $\varepsilon$-function if, for each $y$ in $Y$, there is a neighborhood $D$ of $y$ such that $\operatorname{diam}\left(f^{-1}[D]\right)<\varepsilon$, a one-to-one function between metric spaces is an $\varepsilon$-homeomorphism if it and its inverse are $\varepsilon$-continuous.

Note that any $\varepsilon$-map (i.e., continuous $\varepsilon$-function), defined on a compactum, is a strong $\varepsilon$-function. Note also that $f: X \rightarrow Y$ is a strong $\varepsilon$-function only if every function $g: Y \rightarrow X$, that is a subset of $f^{-1}$, is $\varepsilon$-continuous.

The concept of continua (i.e., connected compacta) being proximately homeomorphic (i.e., $\varepsilon$-homeomorphic, for each positive number $\varepsilon$ ) is probably not fruitful. Perhaps any two continua are proximately homeomorphic. At least, any arc is proximately homeomorphic to any continum. To define an $\varepsilon$-homeomorphism from an $\operatorname{arc} A$ onto any continuum $H$, first decide to map a subarc of $A$ of diameter less than $\varepsilon$ onto all of $H$ except an open set $D$ of diameter less than $\varepsilon$, and then map the rest of $A$ onto $D$. For the first part of the construction, the $\varepsilon$-continuity of the inverse is automatic, and, for the last part, the $\varepsilon$-continuity of the function is automatic. Then it is not difficult (using ideas from the proof of Theorem 1) to see how to get $\varepsilon$-continuity of the function, for the first part of the construction, and $\varepsilon$-continuity of the inverse, for the last part, thus getting an $\varepsilon$-homeomorphism.

Definitions. $f: X \rightarrow Y$ is (proximately) refinable, if, for every positive number $\delta$, there is a $(\delta$-)continuous (strong) $\delta$-function $g: X \rightarrow Y \delta$-near $f$, i.e., such that $d(f(x), g(x))<\delta$, for all $x$ in $X$. $f$ is weakly refinable if $f$ is continuous and, for every positive number $\delta$, there is a $\delta$-continuous function $g: Y \rightarrow X$ such that $g^{-1}$ is $\delta$-near $f \mid g[Y]$, i.e., such that $d(y, f(g(y)))<\delta$, for all $y$ in $Y$. $f$ is a proximate near homeomorphism if, for every positive number $\delta$, there is a $\delta$-homeomorphism $\delta$-near $f$.

It is easy to see that proximately refinable functions are continuous. We could have omitted the $\delta$-continuity condition, on the functions approximating $f$ in the definition of proximate refinability, if we had explicitly required $f$ to be continuous, as we did for weak refinability (see Lemma 2).

3. Inclusion relationships. Refinable maps are proximately refinable, and they in turn are weakly refinable. Refinable maps are proximately refinable, since continuous functions are $\delta$-continuous for all $\delta$, and $\delta$-functions on compacta are strong $\delta$-functions. (If the definition of refinable maps were extended to noncompact domains, the approximating functions should be required to be strong $\delta$-functions.) To see that proximately refinable maps are weakly refinable, let $f: X \rightarrow Y$ be proximately refinable, let $\delta$ be a positive number, and let $G: X \rightarrow Y$ be a $\delta$-continuous, strong $\delta$-function, $\delta$-near $f$. Let $g: Y \rightarrow X$ be a subset of $G^{-1}$. Then $f$ is continuous, $g$ is $\delta$-continuous (since $G$ is a strong $\delta$-function) and $g^{-1}=G \mid g[Y]$ is $\delta$-near $f \mid g[Y]$. Hence $f$ is weakly refinable.

Neither of the inclusions goes the other way, even when the domains are continua.

EXAMPLE 1. A weakly refinable map, on a continuum, that is not proximately refinable.

Let $X$ be the union of the two intervals $A$ and $B$ in the coordinate plane, where $A$ has end points $(0,0)$ and $(1,0)$ and $B$ has end points $(0,1)$ and $(1,0)$. Let $Y=A$, and let $f(a, b)=(a, 0)$, for every $(a, b)$ in $X$. Let $g$ be the identity map on $Y$ (as a map 
into $X$ ). Then $g$ is $\delta$-continuous, and $g^{-1}=f|g[Y]=f| A$ is $\delta$-near $f$, for every positive number $\delta$. Hence $f$ is weakly refinable. But there is no strong $\frac{1}{4}$-function from $X$ onto $Y$ that is $\frac{1}{4}$-near $f$. Hence $f$ is not proximately refinable.

Note that $f \mid A$ is a proximately refinable map onto $Y$. Theorem 4 says this is to be expected when $X$ is a continuum.

Clearly, any proximate near homeomorphism is proximately refinable, so the next example shows that there is a nonrefinable, proximately refinable map defined on a continuum.

EXAMPLE 2. A nonrefinable, proximate near homeomorphism defined on a continuum.

Let $A$ be $[0,1]$ on the $x$-axis in the plane. Let $P_{1}, P_{2}, \ldots$ be a sequence of pseudo-arcs, in the plane, converging to $A$, such that the intersection of any two of $A, P_{1}, P_{2}, \ldots$ is $\{0\}$. Let $X=A \cup\left[\cup_{i=1}^{\infty} P_{i}\right]$, and let $Y=[X \backslash A] \cup\{A\}$ with the decomposition topology. To avoid confusion we use " $a$ " for $A$, when it is considered as a point in $Y$. Let $Q_{i}=\left[P_{i} \backslash A\right] \cup\{a\}$, for $i=1,2, \ldots$ Let $f$ be the projection map from $X$ onto $Y . f$ is clearly not refinable, since there is no 1-map from $X$ onto $Y$ (or even into $Y$ ). This follows from the facts that $A$ has diameter 1 , is mapped onto a locally connected continuum by each continuous function, and $Y$ contains no nondegenerate, locally connected continuum.

Let $\varepsilon$ be a positive number, and let $k$ be a positive integer such that $N_{\varepsilon / 4}(a) \supseteq$ $\cup_{i-k}^{\infty} Q_{i}$. Let $\left\{D_{1}, \ldots, D_{n}\right\}$ be an $\varepsilon / 6$-chain of open disks covering $A$, such that $0 \in D_{1} \backslash D_{2}$. Since $P_{1}, P_{2}, \ldots$ converges to $A$, there is a positive integer $m$ such that $D_{1} \cup \cdots \cup D_{n} \supseteq \cup_{i=m}^{\infty} P_{i}$. Without loss of generality assume $k=m$. Let $g$ be a map from $\cup_{i=m}^{\infty} Q_{i}$ onto $A$ such that $g(a)=0$. Let $C_{1}=\operatorname{cl} D_{1}$, and, for $i=2, \ldots, n$, let $C_{i}=\left[\mathrm{cl} D_{i} \backslash \cup_{j=1}^{i-1} \mathrm{cl} D_{j}\right] \cap\left[A \cup\left(\cup_{i=m}^{\infty} P_{i}\right)\right]$. Assume without loss of generality that $C_{i} \cap A$ has $c$ points, for each $i$. We wish to define a one-to-one $\varepsilon$-homeomorphism $h: X \rightarrow Y$ that is $\varepsilon$-near $f$. Let $h \mid C_{i}$ be a one-to-one function onto $g^{-1}\left[C_{i} \cap A\right]$, for $i=1, \ldots, n$, such that $h(0)=a$. Let $h\left|P_{i}=f\right| P_{i}$, for $i=1, \ldots, m-1$. Let $L=A$ $\cup\left(\cup_{i=m}^{\infty} P_{i}\right)$, and $M=\cup_{i=1}^{m-1} P_{i}$. Then $X=L \cup M$, and $L \cap M=\{0\}$. We wish to show that $h$ is an $\varepsilon$-homeomorphism $\varepsilon$-near $f$, by showing that $h \mid L$ is an $\varepsilon / 2$-homeomorphism $\varepsilon$-near $f$, and observing that $h|M=f| M$ is a homeomorphism. $h \mid L$ is clearly an $\varepsilon / 2$-continuous function $\varepsilon / 2$-near $f$, since $h[L]=f[L] \subseteq$ $N_{\varepsilon / 4}(a) \subseteq N_{\varepsilon / 2}(y)$, for each $y$ in $h[L]$. We now wish to see that the inverse of $h \mid L$ is $\varepsilon / 2$-continuous, by observing that $\left\{g^{-1}\left[D_{1} \cap A\right], \ldots, g^{-1}\left[D_{n} \cap A\right]\right\}$ is a relative open cover of $h[L]$, and showing that $\operatorname{diam}\left(h^{-1}\left[g^{-1}\left[D_{i} \cap A\right]\right]\right)<\varepsilon / 2$, for $i=$ $1, \ldots, n$. For $i=1, \ldots, n$,

$$
g^{-1}\left[D_{i} \cap A\right] \subseteq g^{-1}\left[C_{i-1} \cap A\right] \cup g^{-1}\left[C_{i} \cap A\right] \cup g^{-1}\left[C_{i+1} \cap A\right],
$$

where $C_{0}=C_{1}$ and $C_{n+1}=C_{n}$. Therefore

$$
\begin{aligned}
h^{-1}\left[g^{-1}\left[D_{i} \cap A\right]\right] \subseteq & h^{-1}\left[g^{-1}\left[C_{i-1} \cap A\right]\right] \cup h^{-1}\left[g^{-1}\left[C_{i} \cap A\right]\right] \\
& \cup h^{-1}\left[g^{-1}\left[C_{i+1} \cap A\right]\right]=C_{i-1} \cup C_{i} \cup C_{i+1} .
\end{aligned}
$$

But $\operatorname{diam}\left(C_{i-1} \cup C_{i} \cup C_{i+1}\right)<\varepsilon / 6+\varepsilon / 6+\varepsilon / 6=\varepsilon / 2$. Hence, the inverse of $h \mid L$ is $\varepsilon / 2$-continuous. It follows that $h$ is an $\varepsilon$-homeomorphism $\varepsilon$-near $f$, and, consequently, $f$ is a proximate near homeomorphism. 
In the construction of Example 2, the pseudo-arcs can be replaced by any continua that do not contain arcs.

We now prepare to show that any proximately refinable map, on a compactum with no isolated points, is a proximate near homeomorphism. The next two lemmas follow easily from the definitions.

LEMMA 1. If $f: X \rightarrow Y$ is proximately refinable, then $X$ has an isolated point if and only if $Y$ does.

Lemma 2. If $f: X \rightarrow Y$ is $\varepsilon$-continuous and $g: X \rightarrow Y$ is $\delta$-near $f$, then $g$ is $(2 \delta+\varepsilon)$-continuous.

THEOREM 1. Suppose $f: X \rightarrow Y$ is proximately refinable and $X$ does not have any isolated point. Then $f$ is a proximate near homeomorphism.

Proof. By Lemma 1, $Y$ has no isolated point either. Let $\varepsilon$ be a positive number, and let $g: X \rightarrow Y$ be an $\varepsilon / 3$-continuous, strong $\varepsilon / 3$-function $\varepsilon / 3$-near $f$. Let $\delta$ be a positive number less than $\varepsilon / 3$, such that $\operatorname{diam}\left(g^{-1}[D]\right)<\varepsilon / 3$, if diam $D<\delta$. Such a $\delta$ exists since $Y$ is compact. Let $\left\{D_{1}, \ldots, D_{n}\right\}$ be an open $\delta$-cover of $Y$ such that $D_{i}$ is not contained in $\bigcup_{j=1}^{i-1} \operatorname{cl} D_{j}$, for $i=2, \ldots, n$. Let $C_{1}=\operatorname{cl} D_{1}$, and, for $i=2, \ldots, n$, let $C_{i}=\operatorname{cl} D_{i} \backslash\left(\cup_{j=1}^{i-1} \operatorname{cl} D_{j}\right) . Y$ is the union of the disjoint collection $\left\{C_{1}, \ldots, C_{n}\right\}$. For $i=1, \ldots, n, C_{i}$ contains an open subset of $Y$. Hence $C_{i}$ and $g^{-1}\left[C_{i}\right]$ have $c$ points. Let $h$ be a one-to-one function from $X$ onto $Y$ such that, for $i=1, \ldots, n$, $C_{i}=h\left[g^{-1}\left[C_{i}\right]\right]$.

If $x \in X$, then $g(x) \in C_{i}$, for some $i$, and $h(x) \in C_{i}$ also. Since diam $C_{i}<\varepsilon / 3, h$ is $\varepsilon / 3$-near $g$, and hence, by Lemma $2, h$ is $\varepsilon$-continuous $(2(\varepsilon / 3)+\varepsilon / 3=\varepsilon)$. Also, since $g$ is $\varepsilon / 3$-near $f$ and $\varepsilon / 3+\varepsilon / 3<\varepsilon, h$ is $\varepsilon$-near $f$. Let $y \in Y$. Then $y \in C_{i}$, for some $i$. Let $D^{\prime}$ be an open set of diameter less than $\delta$ containing $C_{i}$, and let $y_{1} \in D^{\prime}$. Then $y_{1} \in C_{j}$, for some $j$. Therefore $h^{-1}(y) \in g^{-1}\left[C_{i}\right] \subseteq g^{-1}\left[D^{\prime}\right]$, and $h^{-1}\left(y_{1}\right) \in g^{-1}\left[C_{j}\right]$. But $\operatorname{diam}\left(g^{-1}\left[D^{\prime}\right]\right)<\varepsilon / 3$, diam $\left(g^{-1}\left[C_{j}\right]\right)<\varepsilon / 3$, and $g^{-1}\left[D^{\prime}\right] \cap$ $g^{-1}\left[C_{j}\right] \neq \varnothing$. Hence $\operatorname{diam}\left(g^{-1}\left[D^{\prime}\right] \cup g^{-1}\left[C_{j}\right]\right)<2(\varepsilon / 3)$, so $d\left(h^{-1}(y), h^{-1}\left(y_{1}\right)\right)<\varepsilon$. Consequently $h^{-1}$ is $\varepsilon$-continuous, and so $h$ is an $\varepsilon$-homeomorphism $\varepsilon$-near $f$. Therefore $f$ is a proximate near homeomorphism.

Theorem 1 is used in [3, Corollary 4] to show that, for continua, proximately refinable maps preserve the property of having the proximate fixed point property for one-to-one functions, for onto functions, and for functions that are one-to-one and onto.

Definition [6]. A continuum $X$ has the proximate fixed point property if, for each positive number $\varepsilon$, there is a positive number $\delta$ such that every $\delta$-continuous function $f: X \rightarrow X$ moves some point $x_{0}$ less than $\varepsilon$, i.e., $d\left(x_{0}, f\left(x_{0}\right)\right)<\varepsilon$.

The next example shows that the condition that $X$ have no isolated point is needed in Theorem 1 , even if $f$ is refinable.

EXAMPLE 3. A refinable map, defined on a compactum, that is not a proximate near homeomorphism.

Let $K$ be a Cantor set and $p_{1}, p_{2}, \ldots$ be a sequence of points, in the complement of $K$, having $K$ as its limiting set. Let $X=K \cup\left\{p_{1}, p_{2}, \ldots\right\}$, and let $Y=(X \backslash K)$ $\cup\{K\}$ with the decomposition topology. To avoid confusion, we use " $k$ " for $K$, 
when it is considered as a point in $Y$. Let $f$ be the decomposition map from $X$ onto $Y$. Clearly, $f$ is not a proximate near homeomorphism, since $X$ has $c$ points and $Y$ has only countably many points.

We wish to show that $f$ is refinable. Let $\varepsilon$ be a positive number, and let $q_{0}$ be a point of $K$. Let $q_{1}, q_{2}, \ldots$ be a sequence, in $\left\{p_{1}, p_{2}, \ldots\right\} \cap f^{-1}\left[N_{\varepsilon / 2}(k)\right]$, converging to $q_{0}$. Let $\left\{D_{1}, D_{2}, \ldots\right\}$ be a disjoint $\varepsilon$-cover of $K \backslash\left\{q_{0}\right\}$ consisting of open and closed subsets of $\left[X \backslash\left\{q_{0}, q_{1}, q_{2}, \ldots\right\}\right] \cap f^{-1}\left[N_{\varepsilon / 2}(k)\right]$, such that $D_{1}, D_{2}, \ldots$ converges to $\left\{q_{0}\right\}$. Assume without loss of generality that $X \backslash\left[\left\{q_{0}, q_{1}, q_{2}, \ldots\right\} \cup\right.$ $\left.\left(\cup_{i=1}^{\infty} D_{i}\right)\right]=\left\{p_{1}, \ldots, p_{n}\right\}$. For $i=1, \ldots, n$, let $g\left(p_{i}\right)=f\left(p_{i}\right)=p_{i}$. For $i=1,2, \ldots$ and $x$ in $D_{i}$, let $g(x)=p_{n+2 i-1}$. Let $g\left(q_{0}\right)=k$, and, for $i=1,2, \ldots$, let $g\left(q_{i}\right)=$ $p_{n+2 i}$. Then $g$ is an $\varepsilon$-map $\varepsilon$-near $f$, so $f$ is refinable.

4. Properties. One of the most useful properties of refinable maps [1, Theorem 1 , p. 264] is also a property of proximately refinable maps, as is stated in Theorem 2. The proof in [1] also works for proximately refinable maps.

THEOREM 2. If $f: X \rightarrow Y$ is proximately refinable and $K$ is a continuum in $Y$, then some component $H$ of $f^{-1}(K)$ is mapped onto $K$ by $f$ and $H \supseteq f^{-1}$ [Int $K$ ].

Weakly refinable maps do not all have the full property stated in Theorem 2, as can be seen in Example 1. They do, however, have the weak confluence part of the property (Theorem 3).

DEFinition. $f: X \rightarrow Y$ is weakly confluent, if some component of $f^{-1}[K]$ maps onto $K$, for each subcontinuum $K$ of $Y$.

The next two lemmas follow easily from the definitions.

DEFINITION [7, p. 13]. If $a$ and $b$ are points, then an $\varepsilon$-chain of points joining $a$ and $b$ is a sequence $a=x_{1}, \ldots, x_{n}=b$ such that $d\left(x_{i}, x_{i+1}\right)<\varepsilon$, for $i=1, \ldots, n-1$.

Lemma 3. Let $f: X \rightarrow Y$ be $\varepsilon$-continuous. Then there is a positive number $\delta$ such that, if there is $a \delta$-chain of points in $X$ joining $x_{1}$ and $x_{2}$, then there is an $\varepsilon$-chain of points in $f[X]$ joining $f\left(x_{1}\right)$ and $f\left(x_{2}\right)$.

LEMMA 4. If (1) $f: X \rightarrow Y$ is $\varepsilon$-continuous, (2) $\delta^{\prime}$ is a positive number such that $\operatorname{diam}(f[D])<2 \varepsilon$, if $\operatorname{diam} D<\delta^{\prime}$, and (3) $g: Y \rightarrow X$ is such that $g^{-1}$ is $\delta$-near $f$, then $\operatorname{diam}\left(g^{-1}[D]\right)<2 \delta+2 \varepsilon$, if diam $D<\delta^{\prime}$, and, hence, $g$ is a strong $(2 \delta+2 \varepsilon)-$ function.

THEOREM 3. If $f: X \rightarrow Y$ is weakly refinable, then it is weakly confluent.

Proof. For $i=1,2, \ldots$, let $g_{i}: Y \rightarrow X$ be a $1 / i$-continuous function such that $g_{i}^{-1}$ is $1 / i$-near $f \mid g_{i}[Y]$. Let $K$ be a continuum in $Y$, and assume without loss of generality that $g_{1}[K], g_{2}[K], \ldots$ converges. Using Lemma 3, it is seen to converge to a continuum $H$.

We wish to show that $f[H]=K$. Let $x_{0} \in H$. Then there are points $y_{1}, y_{2}, \ldots$ in $K$ such that $g_{1}\left(y_{1}\right), g_{2}\left(y_{2}\right), \ldots$ converges to $x_{0}$, and, without loss of generality, $y_{1}$, $y_{2}, \ldots$ converges to a point $y_{0}$ in $K$. Since $f$ is continuous, $f\left(g_{1}\left(y_{1}\right)\right), f\left(g_{2}\left(y_{2}\right)\right), \ldots$ converges to $f\left(x_{0}\right)$. But, for $i=1,2, \ldots, d\left(y_{i}, f\left(g_{i}\left(y_{i}\right)\right)\right)<1 / i$, since $g_{i}^{-1}$ is $1 / i$-near $f$. Hence, $f\left(g_{1}\left(y_{1}\right)\right), f\left(g_{2}\left(y_{2}\right)\right), \ldots$ converges to $y_{0}$. Therefore $f\left(x_{0}\right)=y_{0} \in K$, so $f[H] \subseteq K$. 
Let $\dot{y}_{0} \in K$, and assume without loss of generality that $g_{1}\left(y_{0}\right), g_{2}\left(y_{0}\right), \ldots$ converges. The limit is some $x_{0}$ in $H$. Then $f\left(x_{0}\right)=\lim f\left(g_{i}\left(y_{0}\right)\right)=y_{0}$. Hence, $K \subseteq$ $f[H]$, and so $K=f[H]$. Consequently, the component of $f^{-1}[K]$ that contains $H$ maps onto $K$, so $f$ is weakly confluent.

THEOREM 4. If $f: X \rightarrow Y$ and $Y$ is a continuum, then $f$ is weakly refinable, if and only if there is a continuum $X^{\prime}$ in $X$, such that $f\left[X^{\prime}\right]=Y$, and $f \mid X^{\prime}$ is a proximate near homeomorphism.

Proof. If there is a subcontinuum $X^{\prime}$ of $X$, such that $f\left[X^{\prime}\right]=Y$ and $f \mid X^{\prime}$ is a proximate near homeomorphism, then $f \mid X^{\prime}$ is weakly refinable. That is easily seen to imply that $f$ is weakly refinable.

Assume $f$ is weakly refinable. For every positive number $i$, let $g_{i}: Y \rightarrow X$ be a $1 / i$-continuous function such that $g_{i}^{-1}$ is $1 / i$-near $f$. Assume without loss of generality that $g_{1}[Y], g_{2}[Y], \ldots$ converges. The limit $X^{\prime}$ is closed and, by Lemma 3, is seen to be connected. Hence $X^{\prime}$ is a continuum. By the proof of Theorem 3, $f\left[X^{\prime}\right]=Y$.

Let $\varepsilon$ be a positive number. Let $\delta$ be a positive number less than $\varepsilon / 2$ such that $\operatorname{diam}(f[D])<\varepsilon / 2$ and $\operatorname{diam}\left(g_{i}^{-1}[D]\right)<2 / i+\varepsilon / 2$, for $i=1,2, \ldots$ and $\operatorname{diam} D<\delta$. Such a $\delta$ exists by Lemma 4 . Let $\left\{D_{1}, \ldots, D_{n}\right\}$ be an open (in $X$ ) $\delta / 2$-cover of $X^{\prime}$ such that $D_{1} \cap X^{\prime} \neq \varnothing$ and $D_{i} \cap X^{\prime}$ is not contained in $\bigcup_{j=1}^{i-1} \mathrm{cl} D_{j}$, for $i=2, \ldots, n$. Let $L_{1}=\operatorname{cl} D_{1}$, and, for $i=2, \ldots, n$, let $L_{i}=\operatorname{cl} D_{i} \backslash \cup_{j=1}^{i-1} \operatorname{cl} D_{j}$. Let $k>4 / \varepsilon$ be a positive integer such that $g_{k}[Y] \subseteq \cup_{i=1}^{n} D_{i}$ and $g_{k}^{-1}\left[L_{i} \cap g_{k}[Y]\right]$ contains an open set, for $i=1, \ldots, n$. Let $g=g_{k}$. For $i=1, \ldots, n$, let $C_{i}^{\prime}=L_{i} \cap X^{\prime}$ and let $C_{i}=L_{i}$ $\cap g[Y] . X^{\prime}$ is the union of the disjoint collection $\left\{C_{1}^{\prime}, \ldots, C_{n}^{\prime}\right\}$, and $Y$ is the union of the disjoint collection $\left\{g^{-1}\left[C_{1}\right], \ldots, g^{-1}\left[C_{n}\right]\right\}$. For $i=1, \ldots, n, C_{i}^{\prime}$ and $g^{-1}\left[C_{i}\right]$ contain $c$ points, since each contains an open subset of a continuum.

In order to use the experience gained in proving Theorem 1 , we define an $\varepsilon$-homeomorphism $h: Y \rightarrow X^{\prime}$ whose inverse is $\varepsilon$-near $f \mid X^{\prime}$. Let $h$ be a one-to-one function from $Y$ onto $X^{\prime}$ such that, for $i=1, \ldots, n, h\left[g^{-1}\left[C_{i}\right]\right]=C_{i}^{\prime}$. If $y \in Y$, then $g(y) \in C_{j}$, for some $j$, and hence, $h(y) \in C_{j}^{\prime}$. Since $C_{j} \cup C_{j}^{\prime} \subseteq D_{j}$, and diam $D_{j}<$ $\delta / 2<\varepsilon / 4, h$ is $\varepsilon / 4$-near $g$. By Lemma $2, h$ is $\varepsilon$-continuous, since $g$ is $\varepsilon / 4$-continuous and $2(\varepsilon / 4)+\varepsilon / 4<\varepsilon$. If $x \in X^{\prime}$, then $x \in C_{i}^{\prime}$, for some $i$. Let $D^{\prime}$ be an open set of diameter less than $\delta / 2$ such that $D^{\prime} \subseteq \operatorname{cl} D_{i} \subseteq\left(C_{i} \cup C_{i}^{\prime}\right)$. Let $x_{1} \in D^{\prime} \cap X^{\prime}$. Then $x_{1} \in C_{j}^{\prime}$, for some $j$. Therefore $h^{-1}(x) \in g^{-1}\left[C_{i}\right]$ and $h^{-1}\left(x_{1}\right) \in g^{-1}\left[C_{j}\right]$. But $\left(C_{i} \cup C_{j}\right) \subseteq\left(D^{\prime} \cup \mathrm{cl} D_{j}\right)$ and $\operatorname{diam}\left(D^{\prime} \cup \mathrm{cl} D_{j}\right)<\delta$, so $\operatorname{diam}\left(g^{-1}\left[C_{i} \cup C_{j}\right]\right)<2 / k$ $+\varepsilon / 2<\varepsilon$. Hence $d\left(h^{-1}(x), h^{-1}\left(x_{1}\right)\right)<\varepsilon$, so $h^{-1}$ is $\varepsilon$-continuous.

Let $x \in X^{\prime}$. Then $x \in C_{j}^{\prime}$, for some $j$, and $x_{1}=g\left[h^{-1}(x)\right] \in C_{j}$. Hence

$$
d\left(h^{-1}(x), f\left(x_{1}\right)\right)=d\left(h^{-1}(x), f\left(g\left(h^{-1}(x)\right)\right)\right)<1 / k<\varepsilon / 4,
$$

since $g^{-1}$ is $1 / k$-near $f$. But $d\left(f(x), f\left(x_{1}\right)\right)<\varepsilon / 2$, since $d\left(x, x_{1}\right)<\delta / 2$. Therefore $d\left(h^{-1}(x), f(x)\right)<\varepsilon / 4+\varepsilon / 2<\varepsilon$, so $h^{-1}$ is $\varepsilon$-near $f$. It follows that $f \mid X^{\prime}$ is a proximate near homeomorphism.

Example 3 shows that the condition that $Y$ be a continuum cannot be removed from Theorem 4. However, the following two questions are still unanswered. 
Question 1. In Theorem 4, can the condition that $Y$ is a continuum be replaced by the condition that neither $X$ nor $Y$ has an isolated point?

Question 2. In Theorem 4, can the condition that $Y$ is a continuum be removed, if $X^{\prime}$ is merely stated to be a compactum and $f \mid X^{\prime}$ is merely stated to be a proximately refinable map?

[1, Corollary 1.2, p. 264] generalizes to proximately refinable maps, yielding Lemma 5. The proof in [1] also works here, with our Theorem 2 substituted for Theorem 1 of Heath (Ford) and Rogers.

LEMMA 5. Let $f: X \rightarrow Y$ be proximately refinable and let $Y$ be connected im kleinen at $y$. Then $f^{-1}(y)$ is connected. Hence $f$ is monotone if $Y$ is locally connected.

THEOREM 5. If $f: X \rightarrow Y$ is weakly refinable and $X$ is a graph, then $Y$ is a graph.

Proof. $Y$ is locally connected and has a finite number of components, since $f$ is continuous and $X$ has those properties. If each component of $Y$ is a graph, then $Y$ is a graph. From the definition of weak refinability, it can be seen that each component of $Y$ is the image under $f$ of a component $X_{1}$ of $X$ such that $f \mid X_{1}$ is weakly refinable. Hence assume without loss of generality that $X$ is connected. Then $X^{\prime}$, given by Theorem 4 , is a graph, so we also assume without loss of generality that $f$ is a proximate near homeomorphism. Then $f$ is monotone, by Lemma 5 , since it is proximately refinable. Hence $Y$ is a graph, since any monotone image of a graph is a graph.

\section{REFERENCES}

1. J. Ford and J. W. Rogers, Jr., Refinable maps, Colloq. Math. 39 (1978), 263-269.

2. E. E. Grace, Refinable maps on graphs are near homeomorphisms, Topology Proc. 2 (1977), 129-149.

3. - Refinable maps and the proximate fixed point property, Topology Proc. (to appear).

4. __ Proximately refinable maps and $\theta_{n}^{\prime}$-continua (in preparation).

5. E. E. Grace and Eldon J. Vought, Refinable maps and $\theta_{n}$-continua (in preparation).

6. V. Klee, Stability of the fixed point property, Colloq. Math. 8 (1961), 43-46.

7. G. T. Whyburn, Analytic topology, Amer. Math. Soc. Colloq. Publ., vol. 28, Amer. Math. Soc., Providence, R. I., 1942. 